University of Arkansas, Fayetteville

ScholarWorks@UARK

Industrial Engineering Undergraduate Honors

Theses

Industrial Engineering

\title{
$5-2011$
}

\section{Incorporating vertical travel into non-traditional cross aisles for unit-load warehouse designs}

Kirby A. Clark

University of Arkansas, Fayetteville

Follow this and additional works at: https://scholarworks.uark.edu/ineguht

\section{Citation}

Clark, K. A. (2011). Incorporating vertical travel into non-traditional cross aisles for unit-load warehouse designs. Industrial Engineering Undergraduate Honors Theses Retrieved from https://scholarworks.uark.edu/ineguht/7

This Thesis is brought to you for free and open access by the Industrial Engineering at ScholarWorks@UARK. It has been accepted for inclusion in Industrial Engineering Undergraduate Honors Theses by an authorized administrator of ScholarWorks@UARK. For more information, please contact scholar@uark.edu. 


\section{Incorporating Vertical Travel into Non-Traditional Cross Aisles for Unit-Load Warehouse Designs}

an undergraduates honors thesis submitted to the

Department of Industrial Engineering

University of Arkansas

by

Kirby A. Clark

April 27, 2011

Mentor: Russell D. Meller, Ph. D. Reader: John A. White, Ph. D.

Keywords: Non-Linear Optimization, Warehouse Design, Non-Traditional Aisles, Flying-V, Fishbone, Chebychev Travel 


\begin{abstract}
We propose modifications to the travel-time models for non-traditional warehouse layouts, Flying-V and Fishbone, by incorporating a vertical travel dimension. The Flying-V model determines a cross aisle intersection for every picking aisle, while the Fishbone model determines a single point where the inserted cross aisle intersects the side of the warehouse. The shape and angle of the cross aisle is unrestricted for the Flying-V layout, while the angle is unrestricted for the Fishbone layout. The resulting non-linear optimization models incorporate Chebychev travel within the picking aisles. We compare both the shape of the aisle and the percent improvement over a traditional warehouse with the results found in previous research that ignores vertical travel. We show that the percent improvement diminishes as the height of the rack increases, with Fishbone maintaining a higher percent improvement over Flying-V. We also show that while the shape of Flying- $\mathrm{V}$ can be considerably altered by considering vertical travel, the Fishbone layout often maintains its recommended shape regardless of the height of the rack. We also altered the travel rules for the Flying-V model in order to find simpler designs and obtained near-optimal results. We conclude with recommendations for effective implementation of these two designs.
\end{abstract}

\title{
1. Introduction
}

Warehousing allows a company or organization to meet uncertain demand while the product must be moved and stored, usually without gaining value. Moving and storing inventory adds to the cost of an organization, so efforts both in industry and academia are made to reduce these costs. Research has largely focused on the positioning of the product or the routing of the order-picker in order to mitigate the costs incurred from traveling empty. However, recent research has challenged the very configuration of the rack within storage areas. Gue and Meller questioned two apparent "laws" of warehouse layout: that a cross aisle must be perpendicular to the picking aisles, and that the picking aisles must be parallel [4]. They developed the "Flying-V" layout (presented later in Figure 1) to combat the first assumption and found that the expected travel to a pick decreased by approximately $10 \%$ [4]. The "Fishbone" layout (presented later in Figure 6) was then developed as a response to the second law and found that the expected travel to a pick decreased by as much as $20 \%$, which compares favorably to a bound on the improvement of approximately up $23 \%$ [4]. These models describe a layout with a single, central pickup and deposit location in a unit-load warehouse. 
The savings found by Meller and Gue could provide significant savings to unit-load warehouse operations, given that traveling to a pick occupies a large portion of warehousing costs [1]. Travel savings are offset by a $5-10 \%$ reduction in storage density [4].

As vertical travel was ignored in the original models, we wanted to incorporate this aspect of travel into the model to assess whether the savings found in the original Gue and Meller studies were preserved. Our question became, "What is the impact of vertical travel on the placement of the cross aisle and its percent improvement over the traditional layout?"

To measure impact, we must first analyze the nature of vertical travel. Vertical travel may occur in one of three cases within a warehouse. First, a lift truck may have a single motor and only be able to operate in one direction at a time. We will call this the rectilinear case, where a lift truck must travel to the picking location before being allowed to raise the forks of the truck. The second case will be described as the Chebychev case, where the lift truck is capable of traveling to the pick and raising the forks simultaneously. The third case is a compromise between the first two; for which some portions of the travel permits Chebychev movement and some is limited to rectilinear travel.

How do the different vertical travel cases impact the aisle design? The rectilinear travel case will have a predictable effect on the earlier findings of Meller and Gue. The lift truck may travel vertically only after arriving at the pick location. Therefore, vertical travel is an added constant to any travel route and would not affect the shape of the cross aisle because there is no trade-off in traveling via different routes. Percent improvement would decrease in a predictable manner.

Modeling travel in the Chebychev case would measure travel time as the maximum of the travel time to the pick horizontally or vertically. However, pure Chebychev travel is not applicable (or generally used in practice) because raising or lowering a lift while turning could cause the lift truck to roll.

The Compromise case is the most interesting as we limit Chebychev travel to within the picking aisle (the generally accepted practice). This case may be modeled using equations 
derived for Automated Storage and Retrieval Systems, or AS/RSs [2]. And in this case, because a new variable is incorporated, the shape of the cross aisle may change and the percent improvement is likely to diminish.

The remainder of the paper will address how we incorporated vertical travel into the mathematical models for aisle design. First, we investigate other research involved with nontraditional aisle designs in Section 2. Section 3 will introduce the reader to the mechanics within the Flying-V layout, followed by an explanation of the logic behind our model, results, and conclusions that can be gleaned for this layout. Section 4 addresses the same aspects for the Fishbone layout. The final section, Section 5, ties together our results and provides the practical impact of our work.

\section{Related Literature}

Several papers have been written on the topic of optimizing warehouse aisle layouts. Moder and Thornton [5] recorded space efficiency changes by altering the angle of pallet orientation, although they were more concerned with the ease of access to the pallet. Francis [3] worked to optimize warehouse shape, arranging areas within the facility by minimizing the maximum travel distance. Both models only considered rectilinear travel in a two-dimensional world. White [8], however, considered the travel improvement of radial aisles. His design provided the approximate Euclidean efficiencies after implementing four or six radial aisles.

In practice, inserting traditional cross aisles are only recommended, in large unit-load facilities to benefit dual-command picking and reduce congestion [4]. Inserting a traditional cross aisle into a small to medium facility would result in a significant reduction in the picking space [4]. The perceived limitations of an inserted cross aisle possibly prevented the creation of non-traditional designs despite the conclusions of White. Eventually, however, inserted cross aisles reappeared in the literature with the advent of Gue and Meller's Flying$\mathrm{V}$ and Fishbone layouts [4]. Recent research has further developed the ideas presented by Gue and Meller. Pohl at al. [6, 7] analyzed the benefit of the non-traditional layouts when 
implementing a dual-command picking strategy, where in, they [7] also evaluated the impact of optimal slotting versus distance based slotting strategies and still found a significant travel reduction when implementing Fishbone designs, even with an 20-80 demand curve.

\section{Flying-V}

\subsection{Previous Models of the Flying-V Layout}

The Flying-V aisle design is shown in Figure 1 with examples of travel. Gue and Meller [4] identified three sections within the layout that implemented a different travel route. Section I travel involves going along the bottom cross aisle (with half-width $v$ ) to the picking aisle and traveling "up" to the pick. Section II accesses picks below the inserted cross aisle (with half width $w$ ) by taking the inserted cross aisle and traveling "down" to the pick. Travel to all picks "above" the inserted cross aisle are designated as Section III travel, with the worker traveling along the inserted cross aisle to arrive at the picking aisle.

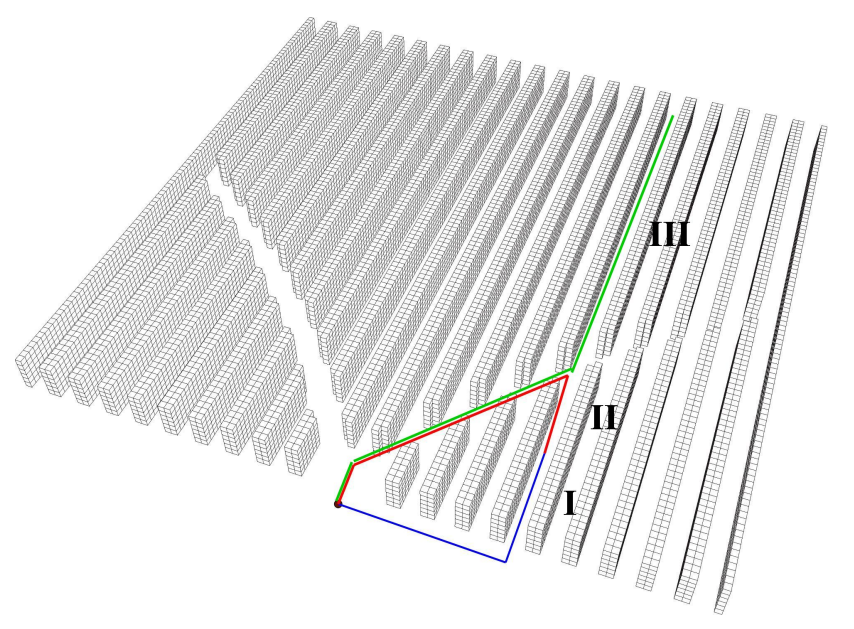

Figure 1: Travel Sections in the Flying-V Layout

Using the above travel rules, the distance to any pick in aisle $i$ can be quickly calculated for a given design. That is, the distance along the inserted cross aisle can be calculated once the points of cross aisle and picking aisle intersection, or $b_{i}$, have been determined. Also note that Gue and Meller specified a break-even relationship that serves as the rule 
for determining whether a pick below the inserted cross aisle is either Section I or Section II travel [4]. The break-even location within the picking aisle was assigned the variable $q_{i}$. The distance to $q_{i}$ using Section I is the distance traveled along the bottom cross aisle plus $q_{i}$. The distance to $q_{i}$, traveling via Section II, is the distance traveled along the inserted cross aisle plus $b_{i}-q_{i}$. Gue and Meller developed an analytical model that minimized the expected travel distance and solved for values of $b_{i}$.

\subsection{Break-Even Travel Time}

In the case without vertical travel, $q_{i}$ defines the point within the picking aisle that an order picker could travel either the bottom cross aisle or the inserted cross aisle and then to the pick with the same travel time. With our incorporation of vertical travel, we need to re-examine the break-even point. The break-even point can be derived by calculating the position within the picking aisle that would yield equivalent travel distances (for ease of exposition, we ignore the width of the inserted cross aisle, $2 w$, for now). Meller and Gue [4] derived the break-even relationship as:

$$
i a+v+q_{i}=v+b_{0}+\sum_{j=1}^{i} d_{j}+b_{i}-q_{i} .
$$

Where, $d_{j}$ represents the distance along the inserted cross aisle between aisle $j-1$ and $j$. Mathematically,

$$
q_{i}=\frac{1}{2}\left[b_{0}+\sum_{j=1}^{i} d_{j}+b_{i}-i a\right]
$$

The value of $q_{i}$ has a lower bound of $b_{i} / 2$ because traveling along the cross aisle is always longer than traveling directly to the picking aisle along the bottom cross aisle. In addition, the value of $b_{i}$ acts as an upper bound on the value of $q_{i}$ (i.e., $q_{i}$ is only valid when less than $\left.b_{i}\right)$

Adding vertical travel to the model introduces a new parameter, $H$, or the number of 
levels of storage in the facility. An indexing convention must also be included, so we will use the term $h_{k}$ to denote the height at level $k$, where $k=0$ for the base level. Travel to the picking aisle remains the same. However, travel within the picking aisle would be determined by the maximum of the vertical and horizontal travel times. Incorporating vertical travel creates the opportunity for the break-even point to change. For a given pick, $h_{k}$ would require the same amount of vertical travel time regardless of the path to the aisle. The break-even point now has the potential to change with each increase of $k$. Furthermore, the value of the break-even point can only increase as vertical travel is included. That is, the break-even point may only increase because Section I has the benefit of longer picking aisle travel to absorb the vertical travel cost. Therefore, we must now implement a break-even value that is sensitive to the change in pick height. That is, we no longer consider $q_{i}$ as the break-even point; we instead define the break-even value for aisle $i$ and level $k$ as $q_{[i, k]}$. Figure 2 illustrates the travel components of a pick in aisle $i$ with $q_{i}$ and $q_{[i, k]}$.

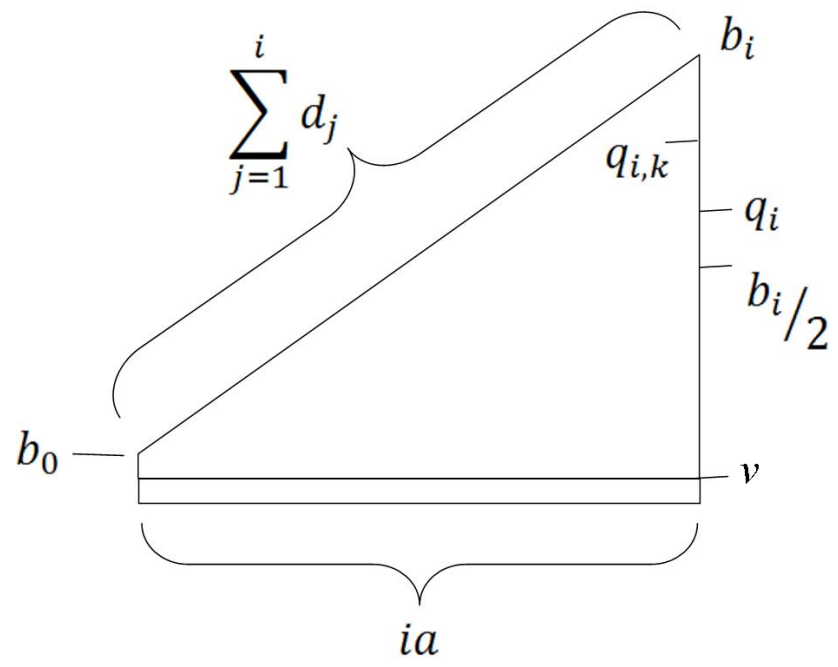

Figure 2: Components of Travel for Section I and II at Height Level $k$

Previous research used travel distances to draw comparisons $[4,6,7]$, but because the drive and lift motors operate at different speeds, a model incorporating vertical travel must use travel times. The minimum travel time (we now add in the inserted cross aisle half width, $w$ ) can be determined from the mathematical expressions of the travel time for the 
two routes. For example, the travel time to some point, $z$, below the inserted cross aisle, is represented as:

$$
\min \left\{\frac{v+i a}{v_{h}}+\max \left\{\frac{z}{v_{h}}, \frac{h_{k}}{v_{v}}\right\}, \frac{v+b_{0}}{v_{h}}+\sum_{j=1}^{i} \frac{d_{j}+w}{v_{h}}+\max \left\{\frac{b_{i}-z}{v_{h}}, \frac{h_{k}}{v_{v}}\right\}\right\}
$$

where the route providing the lower travel time proscribes the best route to $z$. Each route is comprised of the distance to the picking aisle, which is then divided by the horizontal velocity, or $v_{h}$. Time within the picking aisle must be added to each route. As both travel horizontally on the warehouse floor and vertically to reach the pick may be necessary, the expression uses the maximum of the two values. Vertical travel time is determined by the vertical height of the pick, $h_{k}$, and divided by the vertical velocity, $v_{v}$.

In order to better understand how $q_{[i, k]}$ is affected by the height of the pick, a new variable, $x$, may be used. Let $x$ be defined as the horizontal distance that can be traveled during the time to travel a given vertical distance, $h_{k}$. Mathematically,

$$
x=\left(\frac{h_{k}}{v_{v}}\right) v_{h}
$$

Comparing the value of $x$ to a horizontal distance will reveal if the vertical travel will dominate its horizontal counterpart. First, we must consider the extreme cases. That is, what happens when $x$ is less than the shortest horizontal distance in a picking aisle or greater than the longest horizontal distance. Mathematically, this is represented when

$$
\begin{aligned}
& x<b_{i}-w-q_{i} \text {, or } \\
& x>b_{i}-w \text {, respectively. }
\end{aligned}
$$

When $x<b_{i}-w-q_{i}$, horizontal travel time dominates both Section I and II travel. For the purposes of determining a break-even point, vertical travel does not impact the value of $q_{[i, k]}$. Therefore, $q_{[i, k]}=q_{i}$ for all values of $k$. 
When $x>b_{i}-w$, vertical travel time dominates both Section I and II travel. The travel for each section could be modeled as the travel to the aisle plus the vertical travel time. Because the vertical travel time is the same for either section and Section I travel "wins" in a race to the picking aisle, every pick at this height should be made using Section I travel. Therefore, for this case, Section II does not exist (or has a length equal to 0) and $q_{[i, k]}=b_{i}-w$.

Now that the behavior of $q_{[i, k]}$ has been determined for the extreme cases, we must find a value for $q_{[i, k]}$ for the in-between case. This case states that $b_{i}-q_{i} \leq x \leq b_{i}-w$. Because we know that $q_{[i, k]}>q_{i}$, then we know $x>b_{i}-q_{[i, k]}$, which implies that Section II is dominated by vertical travel. An updated break-even equality for this case is then,

$$
\frac{v+i a}{v_{h}}+\frac{q_{[i, k]}}{v_{h}}=\frac{v+b_{0}}{v_{h}}+\sum_{j=1}^{i} \frac{d_{j}+w}{v_{h}}+\frac{h_{k}}{v_{v}} .
$$

Solving for $q_{[i, k]}$ yields

$$
\begin{aligned}
q_{[i, k]} & =b_{0}+w+\sum_{j=1}^{i} d_{j}+\frac{v_{h}}{v_{v}} h_{k}-i a, \\
& =b_{0}+w+\sum_{j=1}^{i} d_{j}+x-i a
\end{aligned}
$$

It is important to note that while logically $q_{[i, k]} \leq b_{i}-w$, mathematically it is possible for $q_{[i, k]}$ to be greater than $b_{i}-w$ in $(6)$. Therefore,

$$
q_{[i, k]}=\min \left\{b_{i}-w, b_{0}+\sum_{j=1}^{i} d_{j}+x-i a\right\}
$$

In order to determine when this occurs, $b_{i}-w$ can be substituted for $q_{[i, k]}$ in the break-even equality and solved for $x$. Mathematically, $q_{[i, k]}=b_{i}-w$ when

$$
x \geq b_{i}-2 w-b_{0}-\sum_{j=1}^{i} d_{j}+i a .
$$


Considering all of the cases, a continuous expression is formulated for $q_{[i, k]}$ :

$$
q_{[i, k]}=\left\{\begin{array}{ll}
q_{[i, 0]}, & x \leq b_{i}-w-q_{[i, 0]} \\
b_{0}+w+\sum_{j=1}^{i} d_{j}+x-i a, & b_{i}-w-q_{[i, 0]}<x<b_{i}-2 w-b_{0}-\sum_{j=1}^{i} d_{j}+i a \\
b_{i}-w, & b_{i}-2 w-b_{0}-\sum_{j=1}^{i} d_{j}+i a \leq x<b_{i}-w \\
b_{i}-w, & x \geq b_{i}-w
\end{array},\right.
$$

which can be reduced to

$$
q_{[i, k]}= \begin{cases}q_{[i, 0]}, & x \leq b_{i}-w-q_{[i, 0]} \\ b_{0}+w+\sum_{j=1}^{i} d_{j}+x-i a, & b_{i}-w-q_{[i, 0]}<x<b_{i}-2 w-b_{0}-\sum_{j=1}^{i} d_{j}+i a \\ b_{i}-w, & x \geq b_{i}-2 w-b_{0}-\sum_{j=1}^{i} d_{j}+i a\end{cases}
$$

\subsection{Model}

Now that we can specify the travel route to any pick in a Flying-V warehouse considering vertical travel, we can model travel in such a warehouse. The first component of travel that must be modeled is travel to the picking aisle. The only difference in the expressions for travel to the aisle from the previous research is that travel time must be calculated instead of distance. The travel time to aisle $i$ for Section I travel is

$$
\frac{v+i a}{v_{h}}
$$

The travel time to aisle $i$ for Section II or III travel is

$$
\frac{1}{v_{h}}\left(v+b_{0}+\sum_{j=1}^{i} d_{j}+w\right) .
$$


Now consider the within aisle travel time (AT) of any pick below the cross aisle, which includes Section I and II. If $q_{[i, k]}=b_{i}-w$, then the expected travel time in aisle $i$ to level $k$ is

$$
E_{i}^{k}[A T \mid I]=\frac{h_{k}}{v_{v}}
$$

because vertical travel dominates. If $q_{[i, k]} \neq b_{i}-w$, then within aisle travel time is based on the time to travel vertically plus the probability that the horizontal travel time will exceed $x$ times the expected horizontal travel time beyond $x$. That is, the expected travel time in aisle $i$ to level $k$ is

$$
E_{i}^{k}[A T \mid I]=\frac{h_{k}}{v_{v}}+\left(\frac{q_{[i, k]}-x}{q_{[i, k]}}\right)\left(\frac{q_{[i, k]}-x}{2 v_{h}}\right) .
$$

Similarly, we can express the expected single command travel time for pick locations in Section II. If $q_{[i, k]}=b_{i}-w$ then Section II does not exist because it has been eliminated from the picking space. Consequently, the probability of traveling to Section II becomes 0 for this case. If $q_{[i, k]} \neq b_{i}-w$ then the expected travel time in aisle $i$ to level $k$ is

$$
E_{i}^{k}[A T \mid I I]=\frac{h_{k}}{v_{v}}+\left(\frac{b_{i}-q_{[i, k]}-w-x}{b_{i}-q_{[i, k]}-w}\right)\left(\frac{b_{i}-q_{[i, k]}-w-x}{2 v_{h}}\right)
$$

Previous research multiplied the expected travel by the probability of a pick in the section to find a total expected travel distance $[4,6]$. However, because Section II can simultaneously exist or not exist in the same picking aisle depending on the height, it is helpful to combine the Section I and II travel times before continuing to total expected travel time. In (16) and (17) we show the expected single-command travel time (including the travel time to the aisle), and in (18) we combine the two terms into one expected travel time for any pick in a 
given aisle $i$ and height $k$ below the cross aisle:

$$
\begin{aligned}
E_{i}^{k}[S C \mid I] & =E_{i}^{k}[A T \mid I]+\left(\frac{v+i a}{v_{h}}\right), \\
E_{i}^{k}[S C \mid I I] & =E_{i}^{k}[A T \mid I I]+\frac{1}{v_{h}}\left(b_{0}+v+\sum_{j=1}^{i} d_{j}+w\right), \\
E_{i}^{k}[S C \mid I+I I] & =E_{i}^{k}[S C \mid I]\left(\frac{q_{[i, k]}}{b_{i}-w}\right)+E_{i}^{k}[S C \mid I I]\left(\frac{b_{i}-q_{[i, k]}-w}{b_{i}-w}\right) .
\end{aligned}
$$

Travel time in aisle $i$ for Section III implements the Chebychev travel-time equations for AS/RSs of Bozer and White [2]:

$$
\begin{aligned}
t_{i}^{I I I}(h) & =\frac{\left(L-b_{i}-w\right)}{v_{h}}, \\
t_{i}^{I I I}(v) & =\frac{H}{v_{v}}, \\
T_{i}^{I I I} & =\max \left\{t_{i}^{I I I}(h), t_{i}^{I I I}(v)\right\}, \\
r_{i}^{I I I} & =\min \left\{\frac{t_{i}^{I I I}(h)}{T_{i}^{I I}}, \frac{t_{i}^{I I}(v)}{T_{i}^{I I I}}\right\}, \\
E_{i}[A T \mid I I I] & =\frac{T_{i}^{I I I}}{2}\left(\frac{\left(r_{i}^{I I I}\right)^{2}}{3}+1\right) .
\end{aligned}
$$

The expected travel time for picks below the cross aisle can be found by averaging the expected travel times for each level $k$. The expected travel time for picks above the cross aisle is simply the addition of the travel to the picking aisle to the expected travel time in the picking aisle for Section III. These statements are represented mathematically as,

$$
\begin{aligned}
E_{i}[S C \mid I+I I] & =\frac{1}{H} \sum_{k=0}^{H} E_{i}^{k}[S C \mid I+I I], \\
E_{i}[S C \mid I I I] & =\frac{1}{v_{h}}\left(b_{0}+\sum_{j=1}^{i} d_{j}+w\right)+\left[\frac{T_{i}^{I I I}}{2}\left(\frac{\left(r_{i}^{I I I}\right)^{2}}{3}+1\right)\right] .
\end{aligned}
$$

Combining (24) and (25) with the appropriate weighting yields the expected travel time to 
retrieve a pick in aisle $i$. This can be expressed as,

$$
\begin{array}{cl}
E_{i}[S C]= & E_{i}[S C \mid I] P(I+I I)+E_{i}[S C \mid I I I] P(I I I), \\
\text { where } \quad & P(I+I I)=\frac{b_{i}-w}{L-2 w}, \text { and } \\
& P(I I I)=\frac{\left(L-b_{i}-w\right)}{L-2 w} .
\end{array}
$$

The total expected travel time in the warehouse is the sum of the expected values for each picking aisle divided by the number of aisles. Mathematically,

$$
E[S C]=\frac{1}{2 n+1}\left(E_{0}[S C]+2 \sum_{i=1}^{n} E_{i}[S C]\right)
$$

\subsection{Results}

The expected travel time model was implemented in Mathematica 7 [9] and optimized using the Nelder-Mead non-linear solver. Warehouse testing ranges were: $H=\{0,1,2,3,4\}, L=$ $\{50,75,100\}, a=5, w=2, v_{h}=50, v_{v}=50 / 6$, and $n=\{9,13,17,21,25,29,33\}$.

In every tested case the performance of the Flying- $V$ layout over the standard layout diminished as the rack height increased, which is illustrated in Figure 3 (where we label $H=0$ as "Bound"). Figure 4 further illustrates that the decline in improvement decreases as the length of the warehouse increases.

In order to compare our results with those of previous research, we re-constructed the two-dimensional model to obtain values for $b_{i}$. The two-dimensional model's values for $b_{i}$ were coded into a calculator to find the percent improvement over a traditional warehouse in a three-dimensional world. The resulting percent improvement would act as a lower bound to the new model, and represented the performance of the previous studies' optimal solutions in a warehouse demanding vertical travel.

Creating the calculator to compute percent improvement sparked a series of ideas. What if the old values for $b_{i}$ were used with the new $q_{[i, k]}$ rules of travel? What would happen 


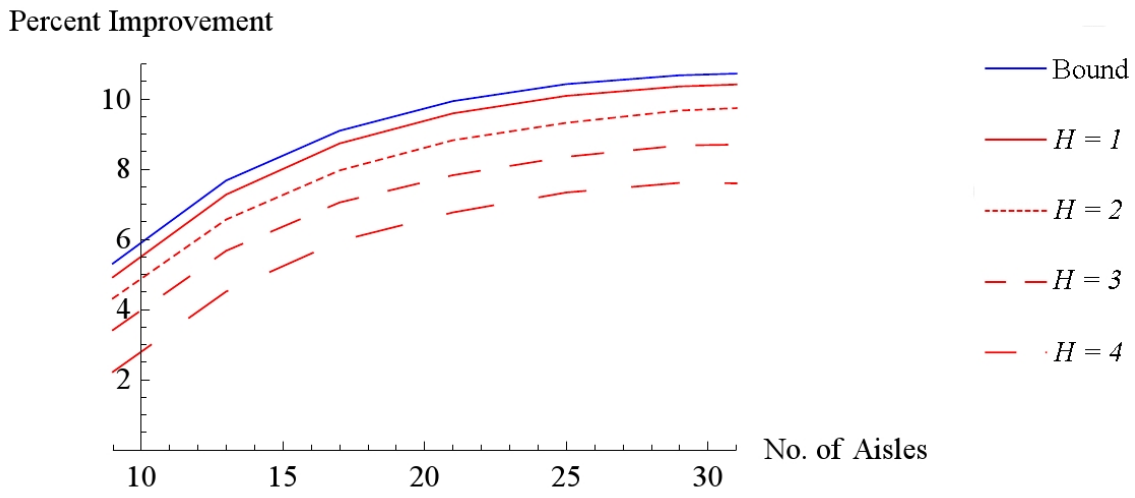

Figure 3: Percent Improvement over Traditional Warehouse of Varied Rack Heights, $L=75$

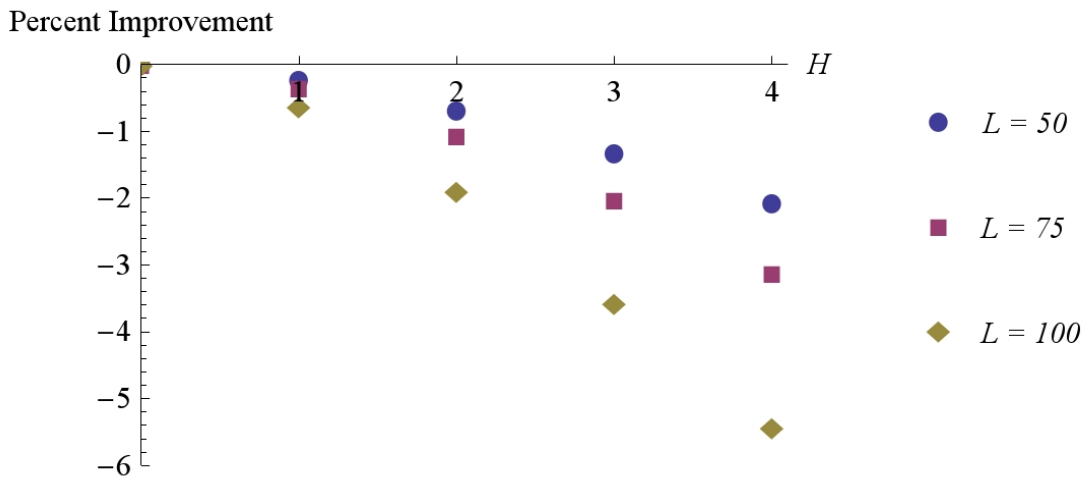

Figure 4: Average Decreased in Percent Improvement (as compared to $H=0$ case) as a Function of Height 
to the percent improvement if Section II travel was no longer an option in our model? In order to answer these questions we tested the same range of warehouse sizes as with our three-dimensional model. The results to be compared are: two-dimensional model in twodimensional space (our upper bound on improvement), three-dimensional model in threedimensional space, two-dimensional model in three-dimensional space using $q_{i}$ (our lower bound), two-dimensional model in three-dimensional using $q_{[i, k]}$, and a model with only Section I and III travel in three-dimensional space.

Figure 5(a) demonstrates typical results found by our model. Percent improvement with-

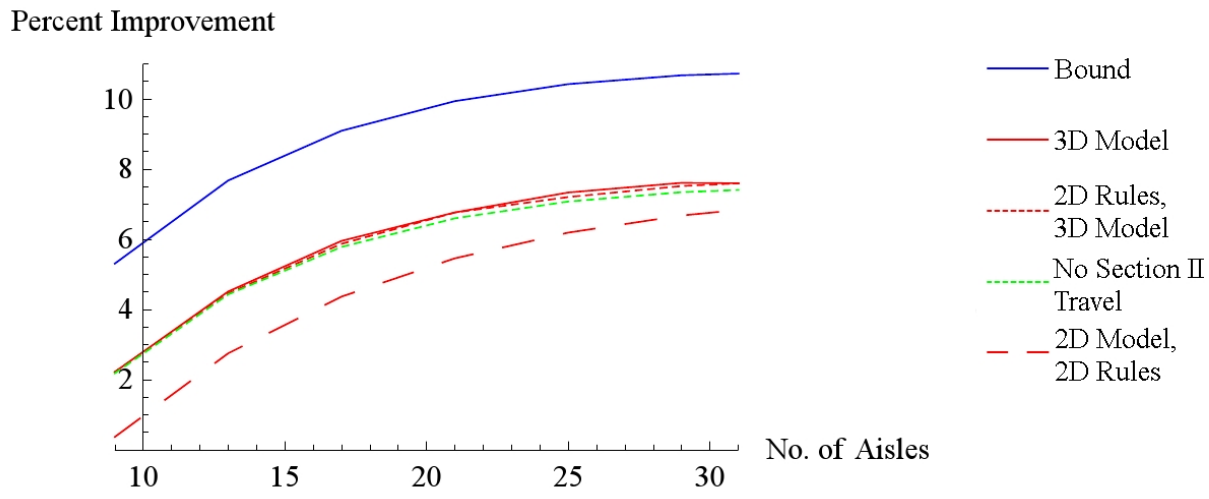

(a) A Comparison of the Models

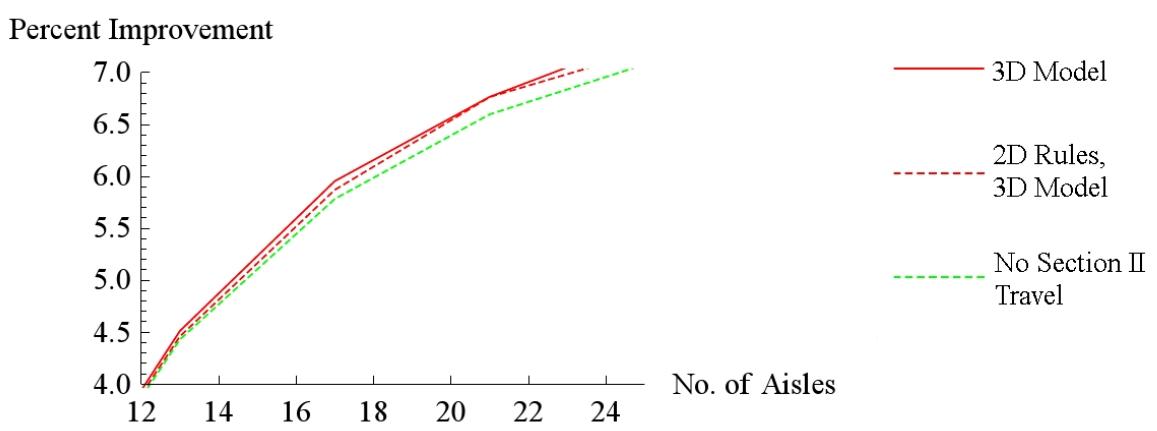

(b) A Closer View of Percent Improvement

Figure 5: Percent Improvement with 5 Levels of Storage and $L=75$

out vertical travel is shown as the bound. We indeed find that incorporating vertical travel to a two-dimensional model results in a significant reduction in the percent improvement, 
as this is the line plotted closest to the bottom of the graph. Three other lines are plotted that are nearly indistinguishable. One of the three is the three-dimensional model in three-dimensional space, which provides the improvements closest to the upper bound. Additionally, the two-dimensional model's values for $b_{i}$ were used to compute the expected three-dimensional travel time with $q_{[i, k]}$ instead of $q_{i}$. The third is a plot of a model that prohibits Section II and optimizes $b_{i}$ values accordingly. A close-up view of the three previously described curves can be found in Figure 5(b).

The change in the value of $b_{i}$ as a function of $H$ is recorded in Table 1 (we have only presented the values for $H=0,2$, and 4 to highlight smaller differences). One can observe that the differences between the values of $b_{i}$ are slight. In fact, we find that, for most cases, the shape or position of the inserted cross aisle is not significantly altered. For some layouts ( $L=50$ and less than 21 picking aisles) the entire inserted cross aisle shifted somewhat closer to the bottom of the warehouse.

\begin{tabular}{|c|c|cc|}
\hline$i$ & $H=0$ & $H=2$ & $H=4$ \\
\hline 1 & 8.16 & 8.15 & 8.10 \\
2 & 13.70 & 13.67 & 13.56 \\
3 & 18.70 & 18.67 & 18.48 \\
4 & 23.25 & 23.23 & 22.94 \\
5 & 27.38 & 27.40 & 27.00 \\
6 & 31.15 & 31.24 & 30.71 \\
7 & 34.59 & 34.73 & 34.10 \\
8 & 37.74 & 37.90 & 37.21 \\
9 & 40.60 & 40.79 & 40.12 \\
10 & 43.22 & 43.40 & 42.75 \\
\hline
\end{tabular}

Table 1: Value of $b_{i}$ as Height Increases, $L=75$

\subsection{Discussion of Results}

The three-dimensional model affirmed that the Flying-V aisle design is advantageous to implement over the standard warehouse configuration. However, that advantage diminishes as the number of levels in the warehouse increases. Our model returned values of $b_{i}$ that, 
overall were approximately the same as those optimized without vertical travel. And in terms of the travel rules associated with our model, based on the $q_{[i, k]}$ values, while these could be coded into the WMS, the software adjustments would take time and money. Given that the model excluding Section II travel gave very similar results, we can see the case for traveling along the base of the warehouse to any picking location under the inserted cross aisle.

\section{Fishbone}

\subsection{Previous Models of the Fishbone Layout}

The Fishbone layout challenges tradition by rearranging the orientation of the picking aisles while incorporating a cross aisle. Figure 6 illustrates the Fishbone concept. Meller and Gue observed that while values for $b_{i}$ could be calculated for every aisle, nearly identical results were produced for a straight-line cross aisle that extends from the P\&D point to a side wall at $b$ [4]. We follow this convention as well. All travel in the Fishbone layout is accomplished through the cross aisle.

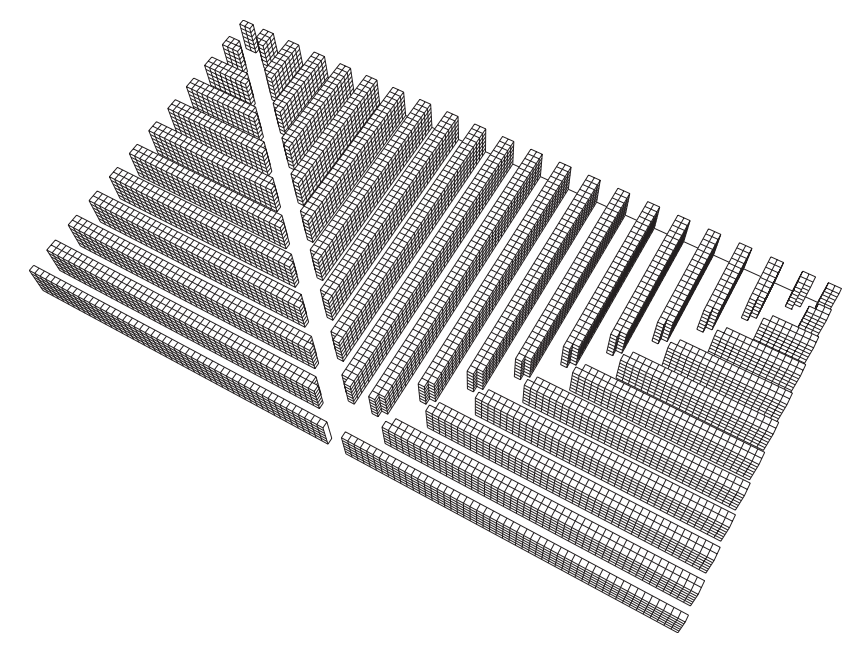

Figure 6: Fishbone Layout 


\subsection{Model}

Travel in a Fishbone warehouse is much simpler than travel in a Flying-V warehouse. Every pick utilizes the V-shaped cross aisle, and no break-even values need to be calculated. Previous research has labeled the two aisle orientations within Fishbone as "horizontal" and "vertical." However, because we are adding a true vertical direction to the model, a new convention is needed. For our purposes, we used \| to represent aisles that are parallel to the base of the warehouse and $\perp$ to represent aisles that are perpendicular to the base of the warehouse. Figure 7 illustrates this convention by the aisles perpendicular to the base in the section labeled II and aisles parallel to the base in sections labeled I, which mirror each other across the perpendicular aisle zero.

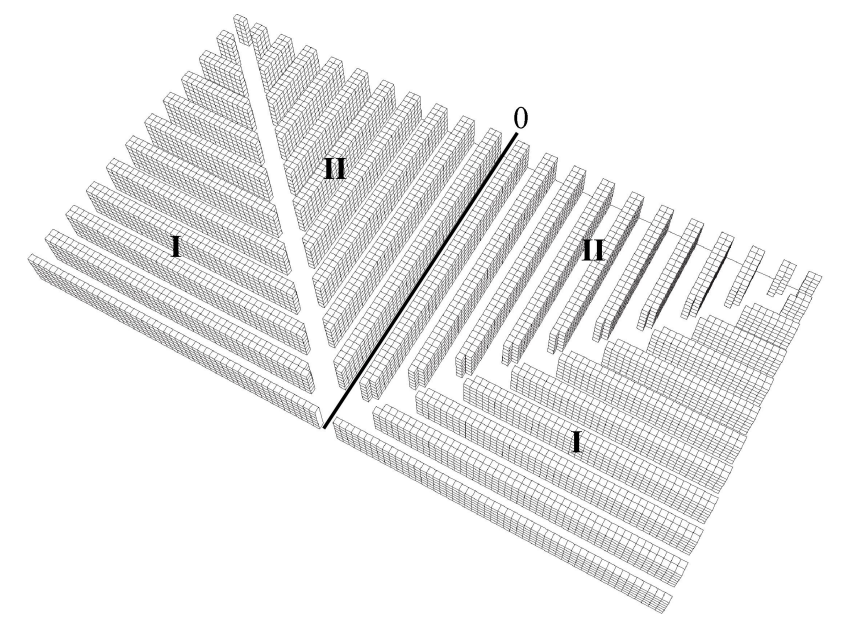

Figure 7: Fishbone Layout

Gue and Meller use the Pythagorean Theorem to determine the distance between picking aisles. Mathematically this is,

$$
d_{\|}=a \sqrt{1+\left(\frac{n a}{b}\right)^{2}}
$$

and

$$
d_{\perp}=a \sqrt{1+\left(\frac{b}{n a}\right)^{2}} .
$$

The lengths of each picking aisle are determined by the amount of space limited by the 
cross aisle and the end of the warehouse. This can be expressed mathematically as

$$
L_{\|, i}=\max \left\{a n-\left(\frac{i n a^{2}}{b}\right)-w, 0\right\},
$$

and

$$
L_{\perp, j}=\max \left\{L-\frac{b j}{n}-w, 0\right\} .
$$

Within aisle travel time for Section I for aisle $i$ can then be expressed using the AS/RS equations:

$$
\begin{aligned}
t_{i}^{I}(h) & =\frac{L_{\|, i}}{v_{h}}, \\
t_{i}^{I}(v) & =\frac{H}{v_{v}} \\
T_{i}^{I} & =\max \left\{t_{i}^{I}(h), t_{i}^{I}(v)\right\}, \\
r_{i}^{I} & =\min \left\{\frac{t_{i}^{I}(h)}{T_{i}^{I}}, \frac{t_{i}^{I}(v)}{T_{i}^{I}}\right\}, \\
E_{i}[A T \mid I] & =\frac{T_{i}^{I}}{2}\left(\frac{\left(r_{i}^{I}\right)^{2}}{3}+1\right) .
\end{aligned}
$$

Within aisle travel time for Section II for aisle $j$ is:

$$
\begin{aligned}
t_{j}^{I I}(h) & =\frac{L_{\perp, j}}{v_{h}}, \\
t_{j}^{I I}(v) & =\frac{H}{v_{v}}, \\
T_{j}^{I I} & =\max \left\{t_{j}^{I I}(h), t_{j}^{I I}(v)\right\}, \\
r_{j}^{I I} & =\min \left\{\frac{t_{j}^{I I}(h)}{T_{j}^{I I}}, \frac{t_{j}^{I I}(v)}{T_{j}^{I I}}\right\}, \\
E_{j}[A T \mid I I] & =\frac{T_{j}^{I I}}{2}\left(\frac{\left(r_{j}^{I I}\right)^{2}}{3}+1\right) .
\end{aligned}
$$

The expected single-command travel time for a pick in Section I is:

$$
E_{i}[S C \mid I]=\left(\frac{i d_{\|}+w}{v_{h}}\right)+\left[\frac{T_{i}^{I}}{2}\left(\frac{\left(r_{i}^{I}\right)^{2}}{3}+1\right)\right] .
$$


The expected single-command travel time for a pick in Section II is:

$$
E_{j}[S C \mid I I]=\left(\frac{j d_{\perp}+w}{v_{h}}\right)+\left[\frac{T_{j}^{I I}}{2}\left(\frac{\left(r_{j}^{I I}\right)^{2}}{3}+1\right)\right]
$$

Only the total amount of picking space is missing in order to determine the probability of each aisle containing a pick. This is facilitated by calculating the picking aisle lengths, where the total of all picking aisle lengths is expressed as,

$$
L_{\text {total }}=2 \sum_{i=0}^{\lfloor b / a\rfloor} L_{\|, i}+L_{\perp, 0}+2 \sum_{j=1}^{n} L_{\perp, j} .
$$

It follows that the total expected travel time in the warehouse is the sum of the expected travel to each individual picking aisle multiplied by the probability of picking in that aisle. Mathematically,

$$
\begin{gathered}
E[S C]=2 \sum_{i=0}^{\lfloor b / a\rfloor} E_{i}[S C \mid I] P(I)+E_{0}[S C \mid I I]\left(\frac{L_{\perp, 0}}{L_{\text {total }}}\right)+2 \sum_{j=1}^{n} E_{j}[S C \mid I I] P(I I) \\
\text { where } P(I)=\frac{L_{\|, i}}{L_{\text {total }}}, \text { and } \\
P(I I)=\frac{L_{\perp, j}}{L_{\text {total }}}
\end{gathered}
$$

\subsection{Results}

Again, we implemented our non-linear travel time model in Mathematica 7 [9]. Parameters for the Fishbone layout were: $H=\{0,1,2,3,4\}, L=\{50,75,100,150\}, a=5, w=2, v_{h}=$ $50, v_{v}=50 / 6$, and $2 n+1=\{5-20\}$.

The Fishbone model calculated the same value for $b$ regardless of the height for almost every scenario we tested. When we decreased the vertical velocity significantly, by an order of magnitude, the value of $b$ was placed close to the base of the warehouse, as expected.

Figure 8 shows the change in performance with each level added to the warehouse. Interestingly, the Fishbone model proved harder for the optimization software to solve, possibly due to the increased use of maximum and minimum functions. 


\section{Percent Improvement}

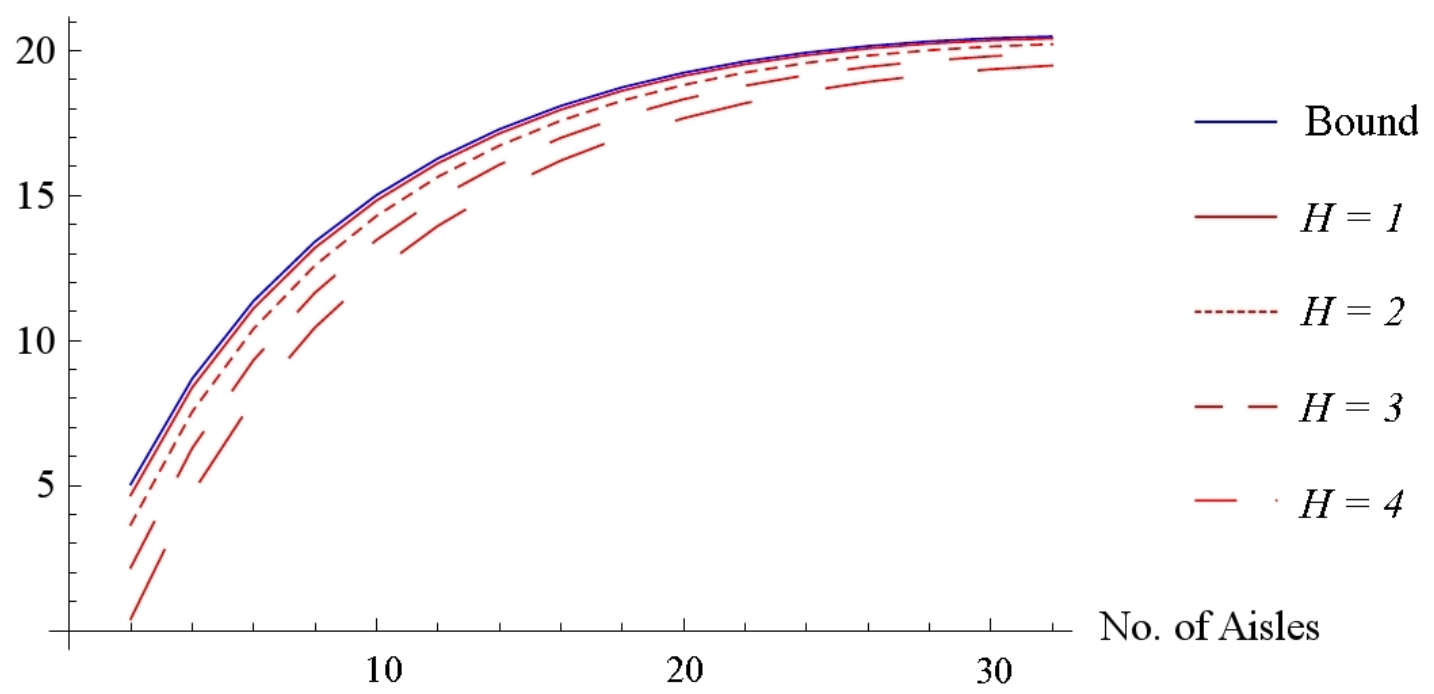

Figure 8: Percent Improvement of Fishbone with Increasing Rack Height, $L=100$

Figure 9(a) and 9(b) show the change in the percent improvement over the traditional warehouse as $L$ increases. In looking at the two graphs, we see that the longer the picking aisle, the more it retains the improvement of its upper bound.

\subsection{Discussion of Results}

Unlike Flying-V, the original travel rules still apply to Fishbone. Additionally, Fishbone retained more of its percent improvement than Flying-V. Because the placement of $b$ remains the same regardless of the rack height, we recommend using the two dimensional model to calculate $b$ with the understanding that the true labor savings will be slightly diminished from what it reports. We observe that there is a significant range over which a warehouse with 5 levels $(H=0)$ will experience at least a $15 \%$ improvement by implementing the Fishbone layout. 


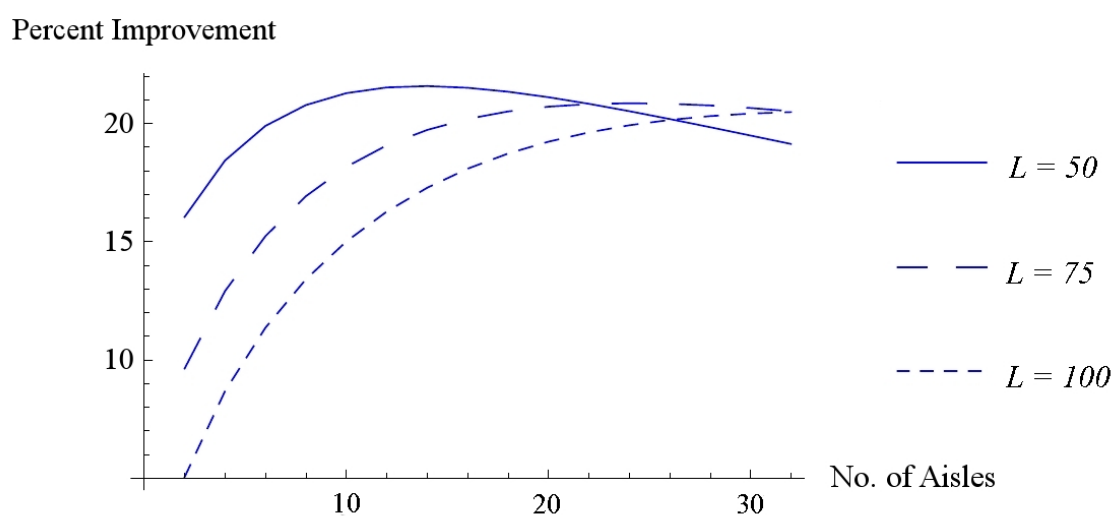

(a) $H=0$

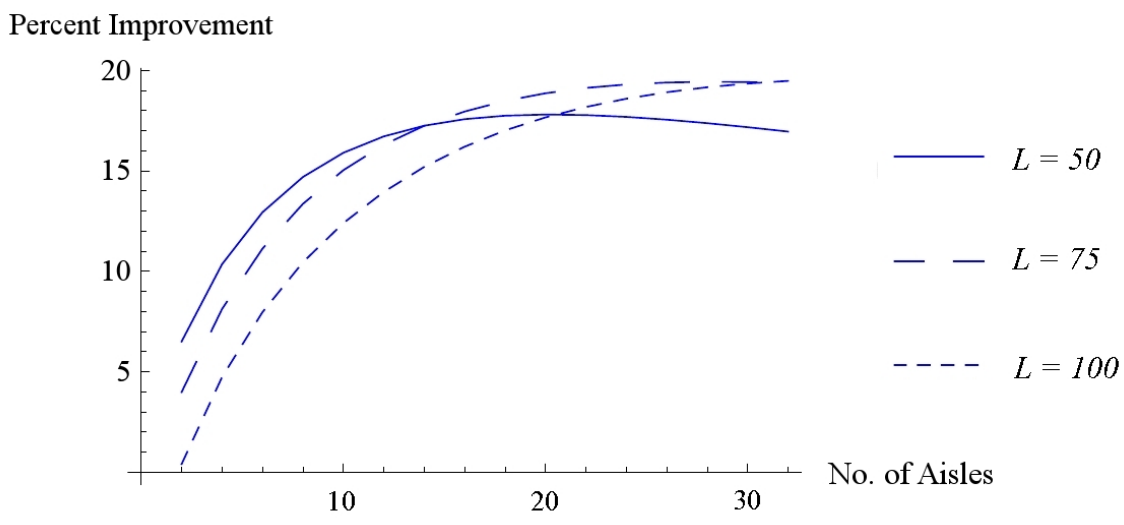

(b) $H=4$

Figure 9: Percent Improvement over Traditional Warehouse with Varied $L$ 


\section{Conclusions}

Previous analytical models found an approximately $10 \%$ improvement for Flying-V and $20 \%$ improvement for Fishbone aisle designs over a standard warehouse layout of the same dimensions [4]. We add fidelity to the model by incorporating vertical travel and we compare both the newly optimized designs and the percent improvement values with the standard layout and the previous research's findings. Our main result is that

As the height of the racking increases, the percent improvement over a traditional layout decreases for both designs.

While previous research suggested that small facilities $(<5$ picking aisles) would not benefit from the non-traditional designs [4], we find that with greater than 3 levels and fewer than 9 picking aisles, converting the layout to a non-traditional design would be detrimental to the travel time.

Another result is that

For both layouts, near-optimal designs are obtained by solving the model ignoring vertical travel.

Our models, which incorporated vertical travel, often took days to solve. However, the optimized aisle design for Fishbone was the same for both models, while the optimized aisle design for Flying-V was similar. This leads to a recommendation,

For both Flying-V and Fishbone layouts, because near-optimal results are obtained using aisle designs optimized while ignoring vertical travel, implement the aisle designs recommended with prior research.

Moreover, because implementing an optimized Flying-V layout requires an organization to code $q_{[i, k]}$ into its WMS, and the results with or without Section II travel are comparable, we recommend

Flying-V layouts with more than 3 levels should ignore Section II travel, accessing all locations under the inserted cross aisle via travel along the bottom cross aisle.

And finally, previous research often compared the percent improvement found with 
Flying- $\mathrm{V}$ and Fishbone designs $[4,6]$. We find that

Fishbone continues to demonstrate a higher expected percent improvement. Moreover, its improvement is affected less by the increase in rack height.

Figures 3 and 8 illustrate the spread of improvement when vertical travel is incorporated into the model. Medium to large warehouses with a Fishbone layout can still expect over a

$15 \%$ travel time reduction over standard warehouse layouts, even with multiple levels.

Future research may incorporate a variety of travel policies that might regain a significant portion of the percent improvement. For instance, the case where a lift truck is allowed to travel with its forks raised within the aisle and lowers the load during its cross aisle travel. The impact of vertical travel might also be considered with dual-command travel. While many options are available to the modeler to develop a model that preserves the percent improvement over traditional layouts, those travel policies must be evaluated with respect to their complexity and the cost of implementation.

In addition, product allocation based on the relative demand of the various products is not as straightforward now that a vertical dimension has been added to the problem. That is, in [7] the product allocation strategy was based strictly on horizontal travel distances. However, if a vertical dimension is also considered, the product allocation strategy would need to be based on travel time. The results of such a strategy would mean that highly-demanded product may continue to be allocated to aisles further out from the pickup and deposit point even as the higher levels of the rack in closer aisles are still unassigned. Developing travel models in such an environment would be an interesting problem left for future research.

\section{References}

[1] Bartholdi, III, J. J., and Hackman, S. T., Warehouse \& Distribution Science, www.warehouse-science.com, 0.87 edition (2008). 
[2] Bozer, Y. A., and White, J. A., "Travel-Time Models for Automated Storage/Retrieval Systems," IIE Transactions, 16, 329-338 (1984).

[3] Francis, R. L., "Sufficient Conditions for Some Optimum-Property Facility Designs," Operations Research, 15, 3, 448-466 (1967).

[4] Meller, R. D., and Gue, K. R., "Aisle Configurations for Unit-Load Warehouses," IIE Transactions, 41, 171-182 (2009).

[5] Moder, J., and Thornton, H., "Quantitative Analysis of the Factors Affecting Floor Space Utilization of Palletized Storage," The Journal of Industrial Engineering, 16, 8-18 (1965).

[6] Pohl, L. M., Meller, R. D., and Gue, K. R., "Optimizing Fishbone Aisles for DualCommand Operations in a Warehouse," Naval Research Logistics, 56, 389-403 (2009).

[7] Pohl, L. M., Meller, R. D., and Gue, K. R., "Turnover-based Storage in Non-Traditional Unit-Load Warehouse Designs," IIE Transactions, Forthcoming.

[8] White, J. A., "Optimum Design of Warehouses Having Radial Aisles," AIIE Transactions, 4, 333-336 (1972).

[9] Wolfram Research, I., "Mathematica Edition: 7.0" (2008). 